\title{
Sulforaphane-N-Acetyl-Cysteine Induces Autophagy Through Activation of ERK1/2 in U87MG and U373MG Cells
}

\author{
Han-Jie Liu ${ }^{a}$ Lei Wang ${ }^{a}$ Lin Kanga,b Juan Du ${ }^{a}$ Sha Li ${ }^{a, b} \quad$ Hui-Xian Cui ${ }^{a, b}$ \\ aDepartment of Human Anatomy, Hebei Medical University, Shijiazhuang, bNeuroscience Research \\ Center, Hebei Medical University, Shijiazhuang, China
}

\section{Key Words}

Glioma $\cdot$ Autophagy $\cdot$ Sulforaphane $\cdot \alpha$-tubulin $\cdot$ Apoptosis

\begin{abstract}
Background/Aims: Sulforaphane- $N$-acetyl-cysteine (SFN-NAC) is a sulforaphane (SFN) metabolite with a longer half-life and better blood-brain barrier permeability than those of SFN. Previous studies have found that SFN-NAC can act via ERK to destroy microtubules and inhibit cell growth in lung cancer cells. However, the underlying mechanisms are unclear, and it is unknown whether SFN-NAC can inhibit the growth of glioma. Here, we have demonstrated for the first time that SFN-NAC activates autophagy-mediated downregulation of $\alpha$-tubulin expression via the ERK pathway. Methods: U87MG and U373MG cells, two widely used glioma cell lines, were utilized in this study. Apoptosis assay, western blot analysis, coimmunoprecipitation, immunostaining, and electron microscopy were used to analyze the effect of SFN-NAC on $\alpha$-tubulin and its interaction with microtube-associated protein 1 light-chain 3 (LC3). Results: SFN-NAC induced cell-cycle arrest in the G2/M phase and dosedependently induced intracellular ERK activation, autophagy, and $\alpha$-tubulin downregulation. These SFN-NAC-induced effects were reversed by inhibiting the ERK pathway with its inhibitor PD98059. U87MG and U373MG cells were transfected with LC3 small interfering RNA, and the subsequent inhibition of autophagy reversed the downregulation of $\alpha$-tubulin by SFNNAC. Furthermore, co-immunoprecipitation experiments and confocal microscopy confirmed that SFN-NAC promotes the binding of LC3 with $\alpha$-tubulin in the cytoplasm. Cell viability experiments demonstrate that SFN-NAC inhibits the growth of U87MG and U373MG cell colonies. Conclusion: These findings suggest that SFN-NAC is a novel potential anti-glioma agent.




\section{Cellular Physiology Cell Physiol Biochem 2018;51:528-542

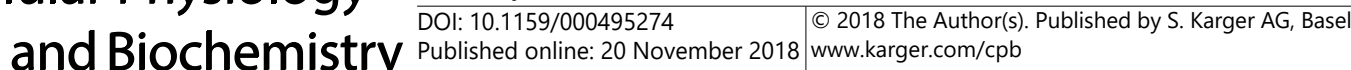 \\ Liu et al.: Sulforaphane-N-Acetyl-Cysteine and $\alpha$-Tubulin}

\section{Introduction}

Glioma is the most common malignant primary tumor of the brain in adults, accounting for $81 \%$ of malignant brain tumors [1], and is categorized into grades I-IV based on its malignant behavior by the World Health Organization (WHO). Classified as a WHO grade IV glioma, glioblastoma multiforme (GBM) is the most common and fatal adult brain tumor and is characterized by a high proliferative rate. Due to the diffuse infiltrating property of gliomas, it is difficult to remove the tumors completely through surgery, and thus residual tumor cells remain, leading to recurrence or disease progression. To date, radiotherapy with concomitant and/or adjuvant chemotherapy is the primary treatment for glioma patients after debulking surgery [2]. However, glioma cells often develop chemotherapy resistance, which results in lower survival rates and shorter survival times [3]. Therefore, new antiglioma chemotherapeutic agents are urgently needed.

Microtubules are basic components of the cytoskeleton of eukaryotic cells and play a key role in the cell cycle and may serve as a therapeutic target for treating glioma [4]. Microtubules are hollow tubes composed of 13 aligned protofilaments assembled from repeating $\alpha$ - and $\beta$-tubulin heterodimers [5]. Microtubules are important in cell biological activities such as the cell cycle [6], intracellular protein transport [7], maintenance of cell morphology [8], cell division [9], and cell metastasis [10]. The $\alpha$-tubulin component of microtubule heterodimers is encoded by at least 15 gene subtypes in the human genome, including TUBA1A, TUBA1B, TUBA1C, TUBA3D, TUBA3E, TUBA4A, and TUBA8 [11]. Different types of $\alpha$-tubulin are expressed at different levels and play different roles in various tissues and biological processes [12-15]. Notably, a recent study showed that increased TUBA1B expression indicates poor prognosis in liver cancer patients [16].

Increasing evidence has shown that the isothiocyanate (ITC) compound sulforaphane (SFN) and its analogs have great potential to prevent the growth of various tumor cells, including those in lung cancer [17], skin cancer [18], colon cancer [19], breast cancer [20], and ovarian cancer [21]. Pharmacokinetic studies in both humans and animals have shown that SFN is metabolized via the mercapturic acid pathway, producing predominantly cysteinylglycine (SFN-CG), cysteine (SFN-Cys), and $N$-acetyl-cysteine conjugates (SFN-NAC), which are excreted in the urine [22]. Sulforaphane (SFN) exists mainly in the form of its derivatives in the circulatory system and tissues. SFN- $N$-acetyl-cysteine (SFN-NAC), an SFN analog, has been detected in central nervous system tissues; it exhibits a longer half-life and a higher plasma concentration than those of SFN and inhibits histone deacetylase (HDAC) activity [23]. Because of its lipophilicity and molecular size, it likely crosses the blood-brain barrier [24]. Furthermore, the presence of SFN could be prolonged by SFN-NAC, which is unstable under physiological conditions and readily dissociates back to SFN [23]. The occurrence of SFN is related to the formation of abnormal mitotic spindles. Previous studies have shown that SFN and its analogs disrupt microtubules in many types of cancer cells. For instance, when the SFN concentration is higher than the minimum effective inhibitory concentration, microtubules are disrupted in human endothelial cells [25], mammary gland adenocarcinoma [26], and lung cancer cells [27]. Recently, SFN and its analogs have been shown to combine covalent and apparently selective microtubules in A549 lung tumor cells [28].

Autophagy is the general intracellular self-defense system, and there are three types: macroautophagy (hereinafter, autophagy), microautophagy, and chaperone-mediated autophagy. The term "autophagy" usually refers to macroautophagy, which is characterized by the formation of double-membraned autophagy vesicles for dysfunctional cellular components, such as misfolded proteins, long-lived proteins, and damaged organelles. These components are sequestered and transported to lysosomes for degradation [29]. Autophagy is an important mechanism for maintaining cellular homeostasis through nutrition recycling and protein quality control. Further evidence has shown that autophagy may be activated during chemotherapy in most tumor cell lines [30]. Autophagy also plays a central role in pharmacological manipulation, and its pathways may serve as therapeutic targets for 
treating cancer [31]. However, the underlying mechanisms remain to be elucidated and it is unclear whether SFN-NAC-induced autophagy occurs in human glioma cells.

In this study, we collected glioma samples from the Chinese Glioma Genome Atlas (CGGA) database (http://www.cgga.org.cn) and The Cancer Genome Atlas (TCGA) network (http:// cancergenome.nih.gov) in order to perform an integrative investigation of the $\alpha$-tubulin gene TUBA1B. We also investigated the effect of SFN-NAC on $\alpha$-tubulin in U87MG and U373MG cells and the underlying mechanisms.

\section{Materials and Methods}

\section{Patients and samples}

The patient information in the publicly accessible CGGA database used in this study was collected from Beijing Tiantan Hospital, in which the Institutional Review Board approved the protocol of this study and informed consent was obtained from each patient. Samples with more than $80 \%$ tumor cells content were selected. Transcriptome sequencing data for glioma samples generated using the Illumina HiSeq platform were obtained from CGGA. Overall survival was estimated from the date of diagnosis until death or the last follow-up date. TCGA mRNA-seq data were downloaded from a public database (https://tcgadata.nci.nih. gov/tcga/tcgaDownload.jsp).

\section{Antibodies and reagents}

Specific antibodies against phosphorylated extracellular signal-regulated kinase (pERK) $1 / 2$, total extracellular signal-regulated kinase (ERK)1/2, microtubule-associated protein 1 light-chain 3 (LC3; Cell Signaling Technology, Danvers, MA), $\alpha$-tubulin (GeneTex, Irvine, CA), and $\beta$-actin (Proteintech, Rosemont, IL) were used for western blot analysis, and antibodies against LC3 (Medical \& Biological Laboratories, Nagoya, Japan) and $\alpha$-tubulin (Santa Cruz Biotech, Santa Cruz, CA) were used for immunofluorescence analysis, confocal microscopy, and co-immunoprecipitation. Additionally, D,L-sulforaphane- $N$-acetyl-L-cysteine

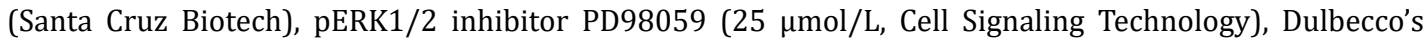
modified Eagle's medium (DMEM)/High glucose culture medium, and fetal bovine serum (FBS) were purchased from HyClone (Logan, UT). Penicillin-streptomycin was obtained from Invitrogen (Carlsbad, CA). Dimethyl sulfoxide was acquired from AppliChem GmbH (Ottoweg4, D-64291 Darmstadt, Germany). Lastly, cOmplete $^{\mathrm{TM}}$ Mini EDTA-free Protease Inhibitor Cocktail (Roche, Basel, Switzerland), BD Cycletest ${ }^{\mathrm{TM}}$ Plus DNA kit (BD Biosciences, Franklin Lakes, NJ), and Lipofectamine ${ }^{\mathrm{TM}}$ RNAiMAX (Invitrogen) were used.

\section{Cell culture and transfection}

The human glioma cell line U87MG was obtained from the Cell Resource Center, Peking Union Medical College (Beijing, China), and U373MG was acquired from the American Type Culture Collection (Manassas, VA). Cells were cultured in DMEM/High glucose culture medium supplemented with 10\% FBS, $100 \mathrm{U} / \mathrm{mL}$ penicillin, and $100 \mathrm{U} / \mathrm{mL}$ streptomycin in a standard humidified incubator under $5 \% \mathrm{CO}_{2}$ at $37^{\circ} \mathrm{C}$. The cells were treated with SFN-NAC for $24 \mathrm{~h}$, transfected with LC3 small interfering RNA (siRNA) for $48 \mathrm{~h}$, and pretreated with the ERK1/2 inhibitor PD98059 (25 $\mu \mathrm{M})$ for $1 \mathrm{~h}$.

To knock down LC3 mRNA transcriptlevels, negative control siRNA( $5^{\prime}$-UUCUCCGAAGGUGUCACGUTT-3') and LC3 siRNA (5'-GAGUGAGCUCAUCAAGAUATT-3') were designed [32]. Cells were plated into 6-well plates at a density of $1 \times 10^{6}$ cells/well and cultured for $24 \mathrm{~h}$. The cells were transfected with the LC3 siRNA or negative control siRNA (30 pmol/well) with Lipofectamine ${ }^{\mathrm{TM}}$ RNAiMAX when the cells reached approximately $60 \%$ confluency. After $24 \mathrm{~h}$ of incubation, the cells were treated with SFN-NAC (30 $\mu \mathrm{M})$ for $24 \mathrm{~h}$.

\section{Cell viability assay}

MTS Cell Proliferation Assay Kits (Promega, Madison, WI) were used to determine cell viability. The cells were seeded at a density of $2 \times 10^{4}$ cells/well in 96-well plates and were incubated with serum-containing media for $24 \mathrm{~h}$. The cells were treated with different concentrations of SFN-NAC $(0-50 \mu \mathrm{M}$ for U87MG, U373MG, and temozolomide-resistant U87MG (U87/TR) cells or $0-90 \mu \mathrm{M}$ for normal human astrocyte (HA) cells for $24 \mathrm{~h}$. The medium was replaced with $100 \mu \mathrm{L}$ of fresh medium, and $20 \mu \mathrm{L}$ of MTS solution was

\section{KARGER}




\section{Cellular Physiology Cell Physiol Biochem 2018;51:528-542

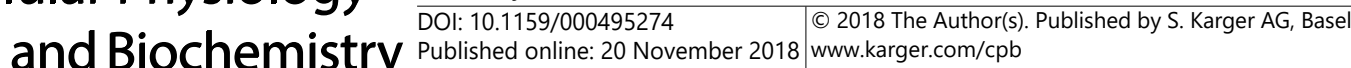 \\ Published online: 20 November 2018 whw kargercom/cph}

added to each well. Blank controls without cells were prepared. Next, $100 \mu \mathrm{L}$ of each sample was transferred to a new 96-well plate and was analyzed with a BioTek Synergy HT Multi-Detection Microplate Reader (BioTek, Winooski, VT). The absorbance values were determined at $490 \mathrm{~nm}$.

\section{Apoptosis assay}

Apoptosis was assessed using an Annexin V-fluorescein isothiocyanate (FITC) propidium iodide (PI) assay. U87MG or U373MG cells were seeded into 6-well plates and treated with SFN-NAC $(30 \mu \mathrm{M})$ or blank medium (negative control) for $24 \mathrm{~h}$. Then, the cells were harvested and centrifuged at 1, $000 \mathrm{rpm}$ for $5 \mathrm{~min}$ and washed twice with cold phosphate-buffered saline (PBS). The cells were re-suspended at a concentration of $1 \times 10^{6}$ cells $/ \mathrm{mL}$ in $100 \mu \mathrm{L}$ of binding buffer and incubated with $5 \mu \mathrm{L}$ V-FITC and $5 \mu \mathrm{L}$ PI for $15 \mathrm{~min}$ at room temperature in the dark. A total of $400 \mu \mathrm{L}$ binding buffer was then added to each tube. All samples were analyzed on a fluorescence-activated cell sorting flow cytometer (FACSAria, BD Biosciences, San Jose, CA) to determine the ratio of apoptotic cells.

\section{Cell cycle assay}

Proliferating U87MG and U373MG cells were exposed to $0 \mu \mathrm{M}$ and $30 \mu \mathrm{M}$ SFN-NAC for $24 \mathrm{~h}$, and the DNA contents were identified using the BD Cycletest ${ }^{\mathrm{TM}}$ Plus DNA kit (BD Biosciences). The cells were harvested after exposure to SFN-NAC for $24 \mathrm{~h}$ and washed three times with ice-cold PBS; the cell concentration of each sample was adjusted to $1.0 \times 10^{6}$ cells $/ \mathrm{mL}$. After sequential incubation without light with an RNase buffer (10 $\mathrm{min})$, followed by incubation with a PI solution (10 $\mathrm{min})$ at room temperature, the DNA content of the cells was analyzed using flow cytometry (BD FACSVerse; BD Biosciences).

\section{Western blot assay}

Cells were lysed using RIPA buffer (APPLYGEN, Beijing, China) containing a protease inhibitor cocktail. A suitable concentration of gel ( $10 \%$ to $15 \%$ sodium dodecyl sulfate-polyacrylamide gel electrophoresis) was selected to separate samples $(30 \mu \mathrm{g})$ containing the same amount of protein, after which the samples were transferred to a nitrocellulose membrane. The membranes were blocked with $1.5 \%$ bovine serum albumin (BSA) in Tris-buffered saline with $0.1 \%$ Tween 20 (TBST) for $1 \mathrm{~h}$ at room temperature, followed by incubation with the primary antibody of the corresponding antigen overnight at $4^{\circ} \mathrm{C}$. After incubation, the membrane was washed with TBST and incubated with fluorescently labeled secondary antibody for $1 \mathrm{~h}$ at room temperature. After three washes in TBST, specific protein bands were detected using the Odyssey infrared imaging system (LI-COR, Lincoln, NE). Additionally, $\beta$-actin was used as an internal control for the loading and standardization of the samples. Each experiment was repeated three times.

\section{Co-immunoprecipitation}

The cells were then treated with SFN-NAC $(30 \mu \mathrm{M})$ for $24 \mathrm{~h}$ after being seeded into 10 -cm cell culture dishes and being cultured for $24 \mathrm{~h}$ at a density of $5 \times 10^{6}$ cells/dish. Next, the cells were washed three times with ice-cold PBS and lysed on ice using a non-denaturing lysis solution containing a protease inhibitor cocktail. Monoclonal anti-alpha-tubulin (Santa Cruz Biotech) or monoclonal anti-LC3 (Medical \& Biological Laboratories) was added to the protein lysate and incubated for approximately $3 \mathrm{~h}$ at $4^{\circ} \mathrm{C}$. The protein $\mathrm{A} / \mathrm{G}$ agarose was then co-incubated overnight, and the purified protein complexes were recovered via centrifugation and heated to $95^{\circ} \mathrm{C}$ for $5 \mathrm{~min}$. The protein complex indicated the binding protein (using western blot analysis).

\section{Immunofluorescence and confocal microscopy}

Cells were seeded at a density of $1 \times 10^{5}$ cells/dish in 35 -mm cover glass-bottom dishes and cultured for $24 \mathrm{~h}$. Then, SFN-NAC was added to a concentration of $30 \mathrm{uM}$. The cells were washed three times with PBS after 24 hours, fixed with 4\% paraformaldehyde at room temperature for $15 \mathrm{~min}$, and treated with $0.5 \%$ Triton X-100 at room temperature for $15 \mathrm{~min}$, after which the solution was discarded. The cells were washed three times with PBS for 3 min each to remove the remaining solution. Next, they were blocked with PBS containing 5\% BSA and $0.1 \%$ Triton X-100 at room temperature for $1 \mathrm{~h}$. The cells were incubated with the primary antibody overnight at $4^{\circ} \mathrm{C}$. The primary antibody was subsequently recovered, and the cells were washed three times for 3 min each with PBS with Tween 20 (PBST). The cells were incubated with the secondary antibody for $1 \mathrm{~h}$ at room temperature. The secondary antibody was then discarded, and the cells 


\section{Cellular Physiology Cell Physiol Biochem 2018;51:528-542

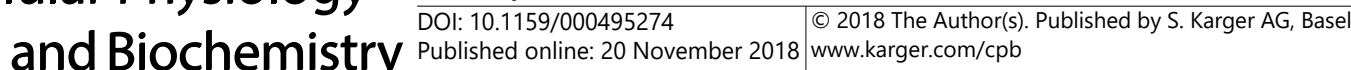

Liu et al.: Sulforaphane-N-Acetyl-Cysteine and $\alpha$-Tubulin

were washed three times for 3 min each with PBST. A 4',6-diamidino-2-phenylindole (DAPI)-containing sealer (ZSGB-BIO, Beijing, China) was then added to the center of each dish. Finally, fluorescence images were observed and photographed using a laser confocal microscope (FV1000, Olympus, Tokyo, Japan).

Transmission electron microscopy

Cells were harvested and fixed with $2.5 \%$ glutaraldehyde at $4^{\circ} \mathrm{C}$ for $3 \mathrm{~h}$. After being washed three times with PBS, the samples were fixed in $1 \%$ osmium tetroxide for $1 \mathrm{~h}$. The samples were then placed in a graded ethanol series consisting of $50 \%, 60 \%, 70 \%, 80 \%, 90 \%$, and absolute ethanol followed by propylene oxide (each stage for 15 to $20 \mathrm{~min}$ ). After dehydration, they were embedded in a 1:1 mixture of acetone and Epon812 resin for $30 \mathrm{~min}$. The samples were then infiltrated in Epon812 for $2 \mathrm{~h}$. Ultrathin sections were cut with a diamond knife, placed on a 200 mesh copper grid, stained with uranyl acetate for $20 \mathrm{~min}$, and then stained with lead nitrate for another $5 \mathrm{~min}$. Immediately thereafter, the samples were rinsed with doubledistilled water and air dried. The sections were examined and photographed using a transmission electron microscope (JEM-2100 Plus, JEOL, Tokyo, Japan).

\section{Statistical analysis}

Paired data were evaluated using Student's $t$-test. The prognostic value of TUBA1B was analyzed using Kaplan-Meier analysis and Cox proportional hazards model analysis using SPSS statistical software (version 19; IBM, Armonk, NY). Statistical analysis was also performed using GraphPad Prism (Version 5; GraphPad Software, San Diego, CA). All data are expressed as the mean \pm standard deviation (SD) from at least three independent experiments. In all statistical tests, $P<0.05$ was considered statistically significant.

\section{Results}

TUBA1B expression enriched in glioblastoma, predicting low survival in glioma patients

To characterize the expression pattern of the $\alpha$-tubulin gene TUBA1B in glioma, we analyzed glioma RNA-sequence data from the CGGA and TCGA databases. Compared with WHO grade II and grade III gliomas, GBM (WHO grade IV) showed the highest expression in the CGGA database (Fig. 1A), which was consistent with the result from TCGA RNA-seq data (Fig. 1B). These results suggest that high expression of TUBA1B is indicative of high-grade glioma. We also analyzed the prognostic value of TUBA1B in a total of 941 glioma patients using the Kaplan-Meier method. As with most malignancies, overexpression of TUBA1B was a significant predictor of poor overall survival in both the CGGA and TCGA databases (Fig. $1 \mathrm{C}$ and D). Furthermore, Cox regression analysis was performed to verify that TUBA1B was an independent prognostic factor in glioma. In the TCGA database (Table 1), univariate Cox analysis showed that TUBA1B, age at diagnosis, WHO grade, and IDH status were significantly associated with overall survival. In multivariate analysis, the expression of TUBA1B was also a significant prognostic factor after adjusting for the clinical factors mentioned above.

\section{SFN-NAC induced cell-cycle arrest and apoptosis}

To clarify the biological role of TUBA1B in glioma, we performed a gene ontology analysis. First, we created a list of the top 600 genes with expression that was strongly correlated with that of TUBA1B through a Pearson correlation analysis based on CGGA and TCGA data. Next, we explored the biological function of these genes through a gene ontology analysis using DAVID Bioinformatics Resources 6.8. When the gene function was sorted by ascending $P$-values, the genes most relevant to TUBA1B were identified to be mostly involved in cell cycle and cell-cycle processes in both CGGA and TCGA databases (Fig. 2A and B).

U87MG and U373MG cells were exposed to $0 \mu \mathrm{M}$ and $30 \mu \mathrm{M}$ SFN-NAC for $24 \mathrm{~h}$. Western blot analysis showed that SFN-NAC downregulated $\alpha$-tubulin in both U87MG and U373MG cells (Fig. 2C). DNA content was analyzed to determine the effect of SFN-NAC on the cell cycle in U87MG and U373MG cells. Compared with the control group, the proportion of cells in the G2/M phase increased significantly after treatment with SFN-NAC in both U87MG and U373MG cell lines (from $12.322 \%$ to $53.350 \%$ in U87MG and from $12.883 \%$ to $51.680 \%$ in U373MG), 


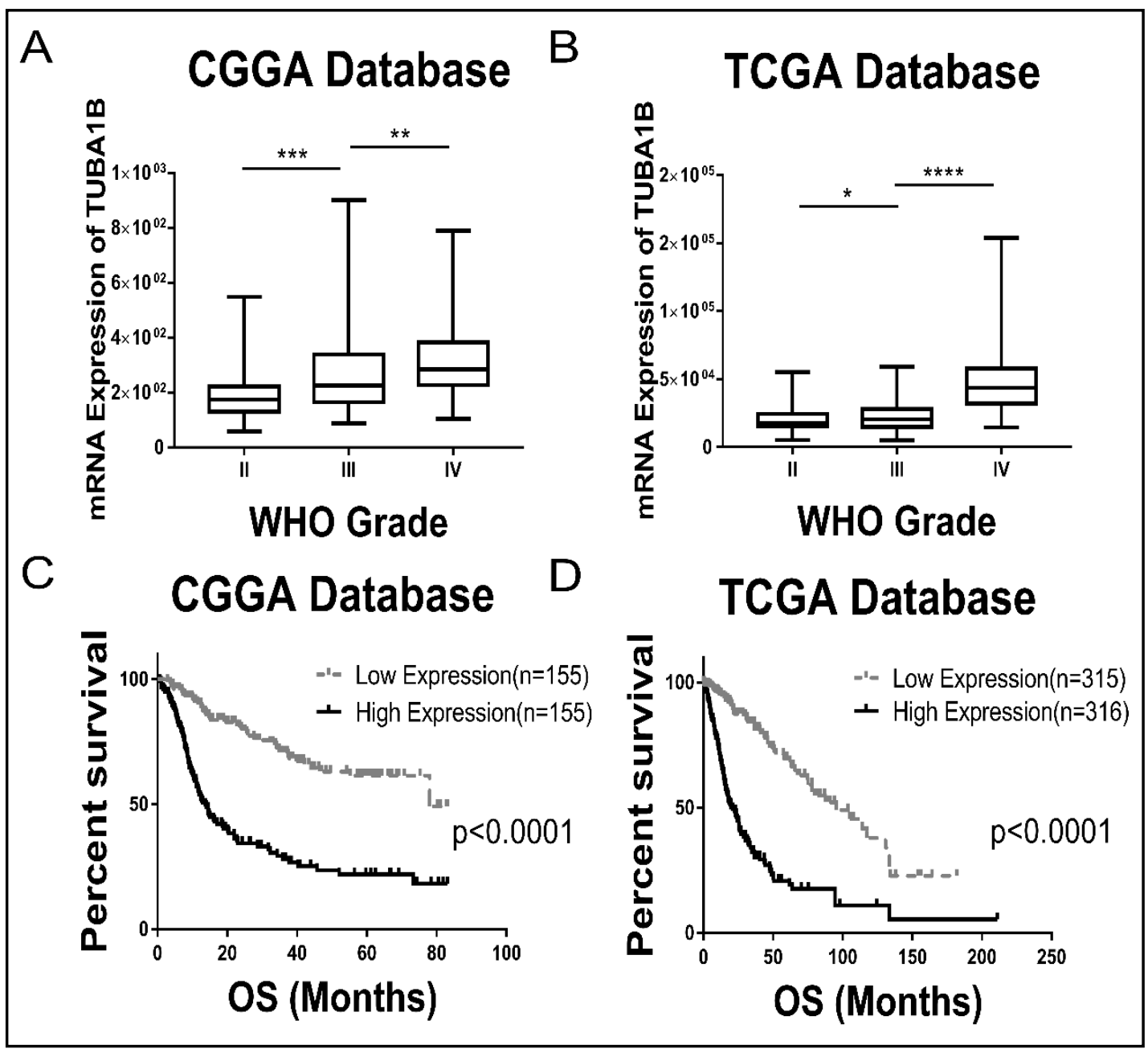

Fig. 1. TUBA1B mRNA expression increased with glioma grade, and it is a prognostic factor in glioma patients. (A, B) TUBA1B mRNA expression was significantly increased in glioblastoma (WHO grade IV) in the CGGA and TCGA datasets. (C, D) Kaplan-Meier survival analysis showed that a high TUBA1B mRNA expression was associated with a shorter survival prognosis in glioma patients.

while the cells in the G1/ G0 phase were significantly decreased (from 56.969\% to $11.407 \%$ in U87MG and from $51.344 \%$ to $21.393 \%$ in U373MG), and the cell distribution change in the $\mathrm{M}$ phase (from $30.709 \%$ to $34.785 \%$ in U87MG and from $35.773 \%$ to $27.927 \%$
Table 1. Univariate and multivariate COX analysis of TUBA1B in TCGA database

\begin{tabular}{lcccc}
\hline \multirow{2}{*}{ Variable } & \multicolumn{2}{c}{ Univariate analysis } & \multicolumn{2}{c}{ Multivariate analysis } \\
& HR $(95 \% \mathrm{Cl})$ & $\mathrm{p}$ Value & HR $(95 \% \mathrm{CI})$ & $\mathrm{p}$ Value \\
\hline TUBA1B Expression & $28.228(16.089-49.526)$ & $<0.0001$ & $3.797(1.847-7.804)$ & $<0.0001$ \\
Diagnosis Age & $1.073(1.061-1.084)$ & $<0.0001$ & $1.043(1.030-1.056)$ & $<0.0001$ \\
WHO Grade & $4.794(3.798-6.051)$ & $<0.0001$ & $1.841(1.360-2.492)$ & $<0.0001$ \\
IDH Status & $0.124(0.090-0.170)$ & $<0.0001$ & $0.424(0.276-0.651)$ & $<0.0001$ \\
\hline
\end{tabular}

in U373MG) was not statistically significant ( $\mathrm{P}>.05$, Fig. 2D). A flow cytometry assay was used to determine the apoptosis ratio in U87MG and U373MG cells. Compared with the control group, the total apoptosis ratio was significantly increased after SFN-NAC treatment in both U87MG and U373MG cells. These results showed that SFN-NAC downregulated $\alpha$-tubulin in glioma cells, thus inducing cell-cycle arrest in the G2/M phase and triggering apoptosis at the same time. 


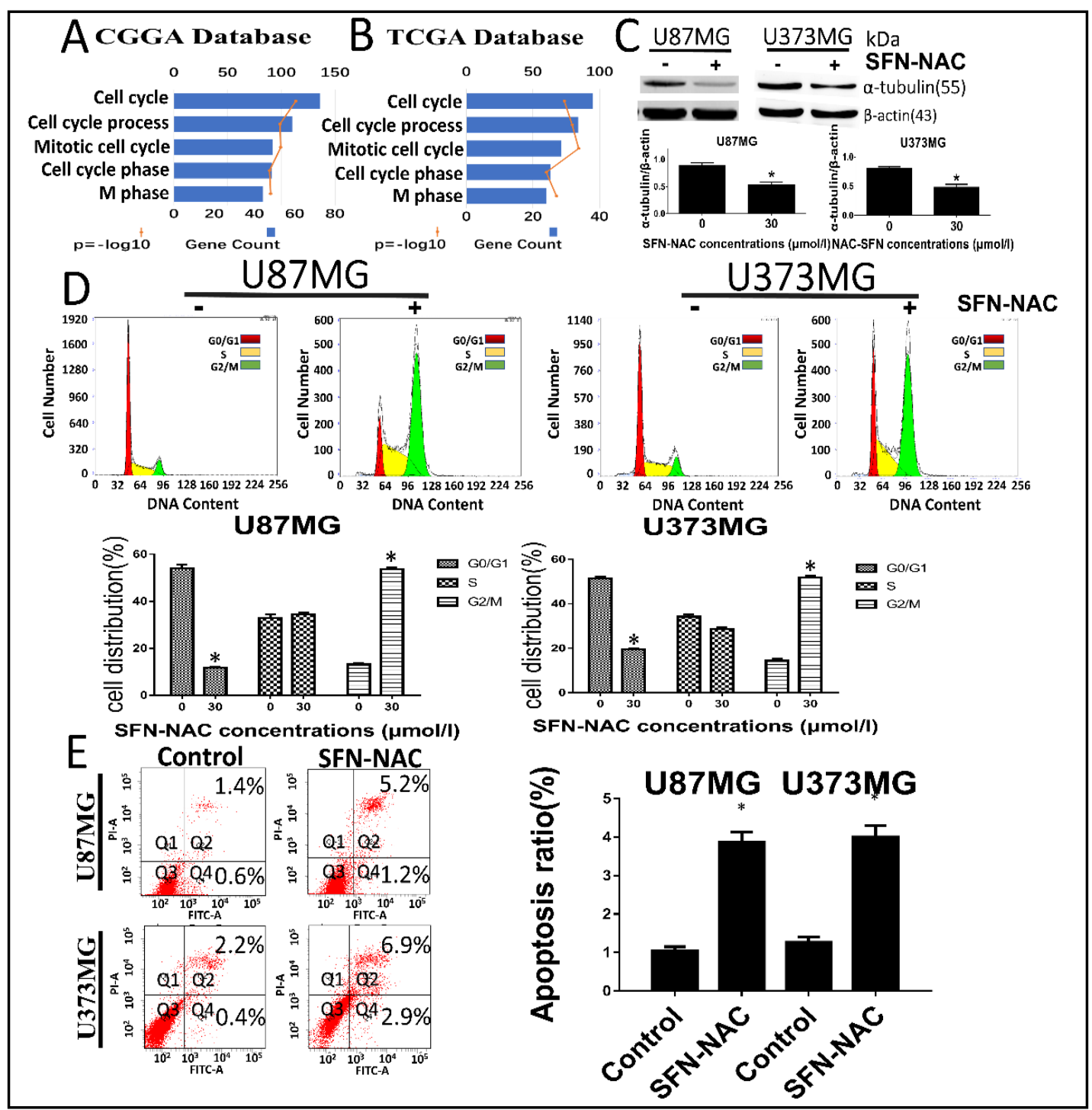

Fig. 2. TUBA1B mRNA was closely related to cell-cycle function in glioma, and SFN-NAC downregulated the expression of $\alpha$-tubulin and induced cell cycle arrest and apoptosis. (A, B) GO analysis showed that the TUBA1B mRNA was primarily involved in cell cycle and cell-cycle processes according to CGGA and TCGA expression data. (C) U87MG and U373MG cells were treated with SFN-NAC (0 $\mu \mathrm{M}$ or $30 \mu \mathrm{M})$ for $24 \mathrm{~h}$, and western blot analysis was performed to assay $\alpha$-tubulin expression, with $\beta$-actin used as a control. (D) U87MG and U373MG cells were treated with SFN-NAC $(0 \mu \mathrm{M}$ or $30 \mu \mathrm{M})$ for $24 \mathrm{~h}$. DNA-content-based analysis of the cell cycle was conducted using flow cytometry and the BD Cycletest ${ }^{\mathrm{TM}}$ Plus DNA kit. (E) U87MG and U373MG cells were treated with SFN-NAC $(0 \mu \mathrm{M}$ or $30 \mu \mathrm{M})$ for $24 \mathrm{~h}$, and the apoptosis ratio was determined by Annexin V/PI assay. The percentage of apoptotic cells is shown at the right side of each flow cytometry image. Data are presented as the mean \pm SD from three separate experiments, with * indicating $\mathrm{P}<0.05$ compared to the control.

SFN-NAC downregulated $\alpha$-tubulin by increasing the LC3-II/LC3-I ratio and activating ERK1/2

U87MG and U373MG cells were treated with different concentrations of SFN-NAC $(0,10$, $20,30,40$, and $50 \mu \mathrm{M}$ ) for $24 \mathrm{~h}$. Western blot analysis showed that SFN-NAC activated ERK1/2 (Thr202/Tyr204), downregulated $\alpha$-tubulin, and induced autophagy in a dose-dependent manner. The ratio of pERK1/2 or cytosolic LC3-II/LC3-I was significantly increased at 30 $\mu \mathrm{M}$ SFN-NAC, and $\alpha$-tubulin was remarkably decreased at the same concentration (Fig. 3A). Therefore, we chose $30 \mu \mathrm{M}$ SFN-NAC as an optimal concentration for the follow-up 

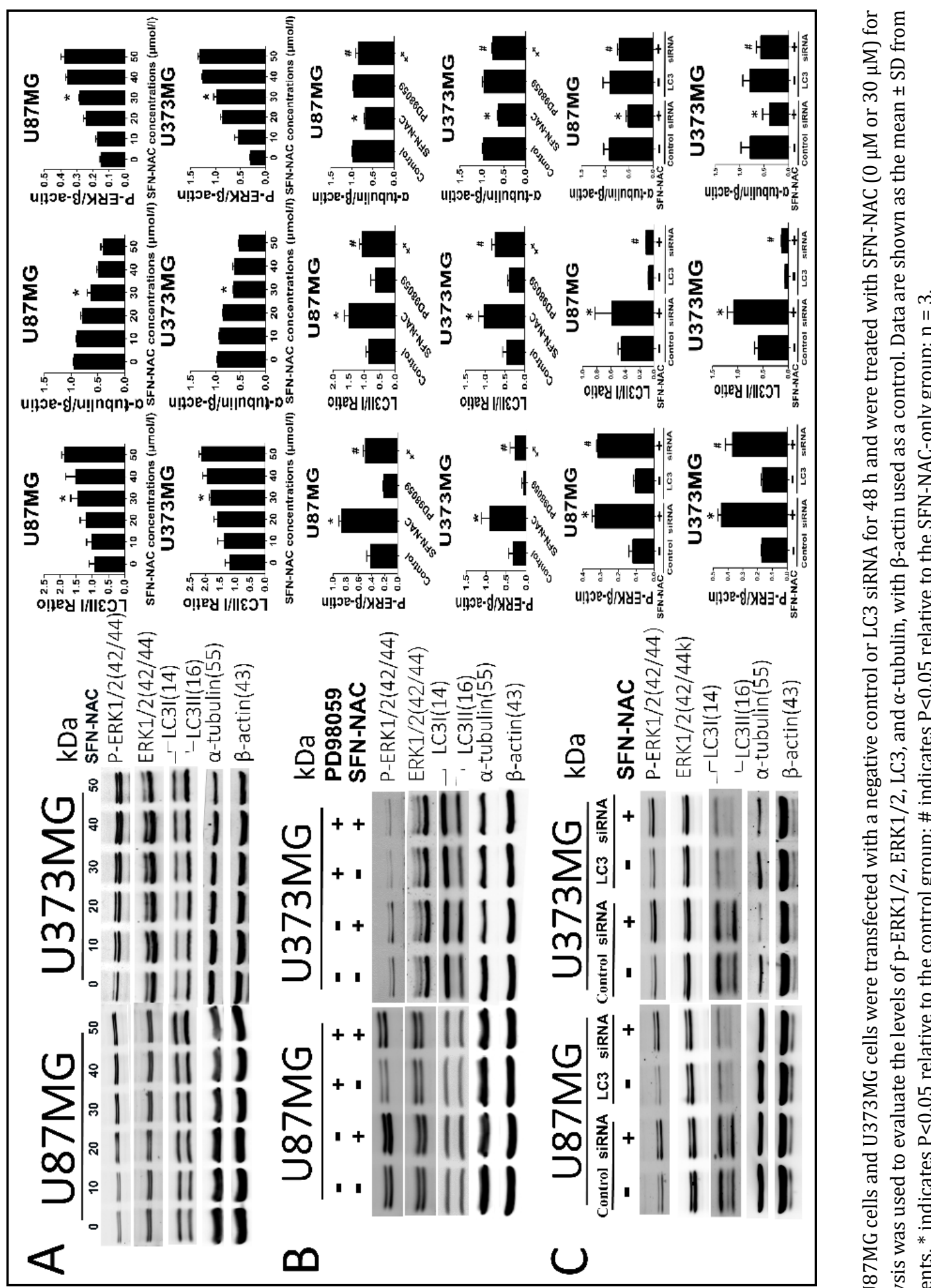

还

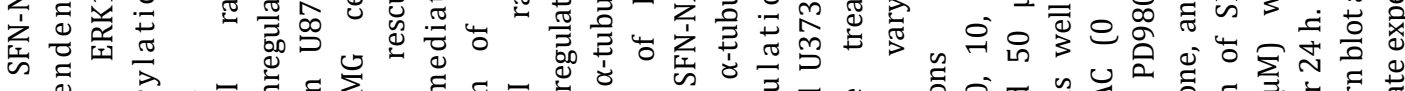
ఏ m बे d

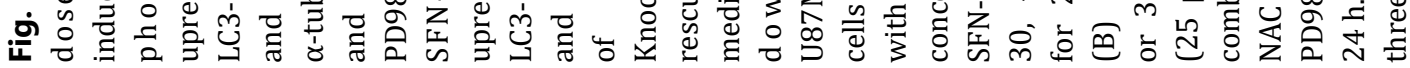
KARGER 
experiments. The LC3-II/LC3-I ratio and the $\alpha$-tubulin level were both altered in the SFNNAC-treated cells, while the increase in the LC3-II/LC3-I ratio and $\alpha$-tubulin downregulation were both rescued by PD98059 (Fig. 3B), which blocks the ERK1/2 pathway, in cells treated with SFN-NAC and PD98059. This result indicated that SFN-NAC induced cell autophagy through activation of ERK1/2, and both LC3 and $\alpha$-tubulin were the downstream effectors of ERK1/2. After the cells were transfected with LC3 siRNA for $48 \mathrm{~h}$ and then treated with SFN-NAC for $24 \mathrm{~h}$, the expression of LC3 was significantly downregulated compared with the untransfected cells; the expression of $\alpha$-tubulin was restored in both LC3-siRNA-transfected cells and SFN-NAC-treated cells compared to that in cells treated with SFN-NAC alone (Fig. 3C). This result suggested that SFN-NAC subsequently downregulated $\alpha$-tubulin through LC3 expression in the U87MG and U373MG cells. Together, these results indicated that SFNNAC activated ERK1/2, increased the LC3-II/LC3-I ratio, and downregulated $\alpha$-tubulin by increasing the LC3-II/LC3-I ratio and activating ERK1/2 in U87MG and U373MG cells.

\section{SFN-NAC enhanced binding between LC3-II and $\alpha$-tubulin and induced autophagy in} U87MG and U373MG cells

We used endogenous co-immunoprecipitation to verify the binding of LC3/ $\alpha$-tubulin after the cells were treated with SFN-NAC. After the cells were treated with $0 \mu \mathrm{M}$ or $30 \mu \mathrm{M}$ SFNNAC for $24 h$, anti- $\alpha$-tubulin was immunoprecipitated with a conjugate of LC3 and $\alpha$-tubulin in either U87MG or U373MG cells. The amount of LC3-II co-immunoprecipitated from the SFNNAC-treated cells was more than that from the untreated cells, suggesting that LC3-II bound to $\alpha$-tubulin and that SFN-NAC significantly increased the binding. Simultaneously, a reverse co-immunoprecipitation assay was performed, revealing that anti-LC3 immunoprecipitated with both LC3 and the $\alpha$-tubulin protein interacting with LC3 (Fig. 4A). These results indicated that SFN-NAC increased the interaction between LC3-II and $\alpha$-tubulin. Further, to investigate the effect of SFN-NAC on microtubules and autophagosomes, we examined and observed the form of microtubules and autophagosomes in U87MG and U373MG cells via an immunofluorescence assay and confocal microscopy. The cells were treated with $0 \mu \mathrm{M}$ and $30 \mu \mathrm{M}$ SFN-NAC for $24 \mathrm{~h}$, stained with an anti- $\alpha$-tubulin fluorescent antibody (green) and an anti-LC3 fluorescent antibody (red), and observed using a fluorescence microscope. The results showed that the microtubules were filamentous and homogeneously formed; the LC3 punctation was darker and dispersed in the cytoplasm in the control group. In the SFN-NAC-treated group, the cell tentacles disappeared, the microtubules were disrupted, the fluorescence intensity was weaker, the shapes of microtubules were contracted, the LC3 punctation was brighter and accumulated, and microtubule disruption was observed in the spaces surrounded by the LC3 punctation. In addition, the overlapping areas (yellow) were brighter in the treated cells than those in the control cells (Fig. 4B). The colocalization of LC3 and $\alpha$-tubulin was promoted in the cells treated with SFN-NAC, which reflected the interaction between LC3 and $\alpha$-tubulin observed in the co-immunoprecipitation assay. Transmission electron microscopy was used to observe the cell ultrastructures. Autophagosomes and autophagolysosomes were found in the U87MG and U373MG cells treated with SFNNAC. In contrast, the cells without SFN-NAC treatment exhibited rare autophagosomes or autophagolysosomes. (Fig. 4C) Therefore, we concluded that SFN-NAC induced autophagy and downregulated $\alpha$-tubulin through autophagy by activating ERK1/2 in the U87MG and U373MG cells.

\section{SFN-NAC decreased glioma cell viability}

To assess the effect of SFN-NAC on cell viability, we examined the antiproliferative effect of SFN-NAC in various normal human astrocyte cells (HA), temozolomide-resistant U87MG cells (U87/TR), U87MG cells, and U373MG cells using an MTT assay. The cells were treated with SFN-NAC at different concentrations for $24 \mathrm{~h}$, and SFN-NAC decreased the cell viability of these cell lines in a dose-dependent manner. The $50 \%$ inhibitory concentrations $\left(\mathrm{IC}_{50}\right.$ ) of SFN-NAC in each cell line were $60.08 \mu \mathrm{M}$ for HA, 35.20 $\mu \mathrm{M}$ for U87MG, 39.11 $\mu \mathrm{M}$ for U373MG, and $36.20 \mu \mathrm{M}$ for the U87/TR cells following SFN-NAC treatment for $24 \mathrm{~h}$ (Fig. 5A, B, C, and

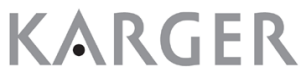




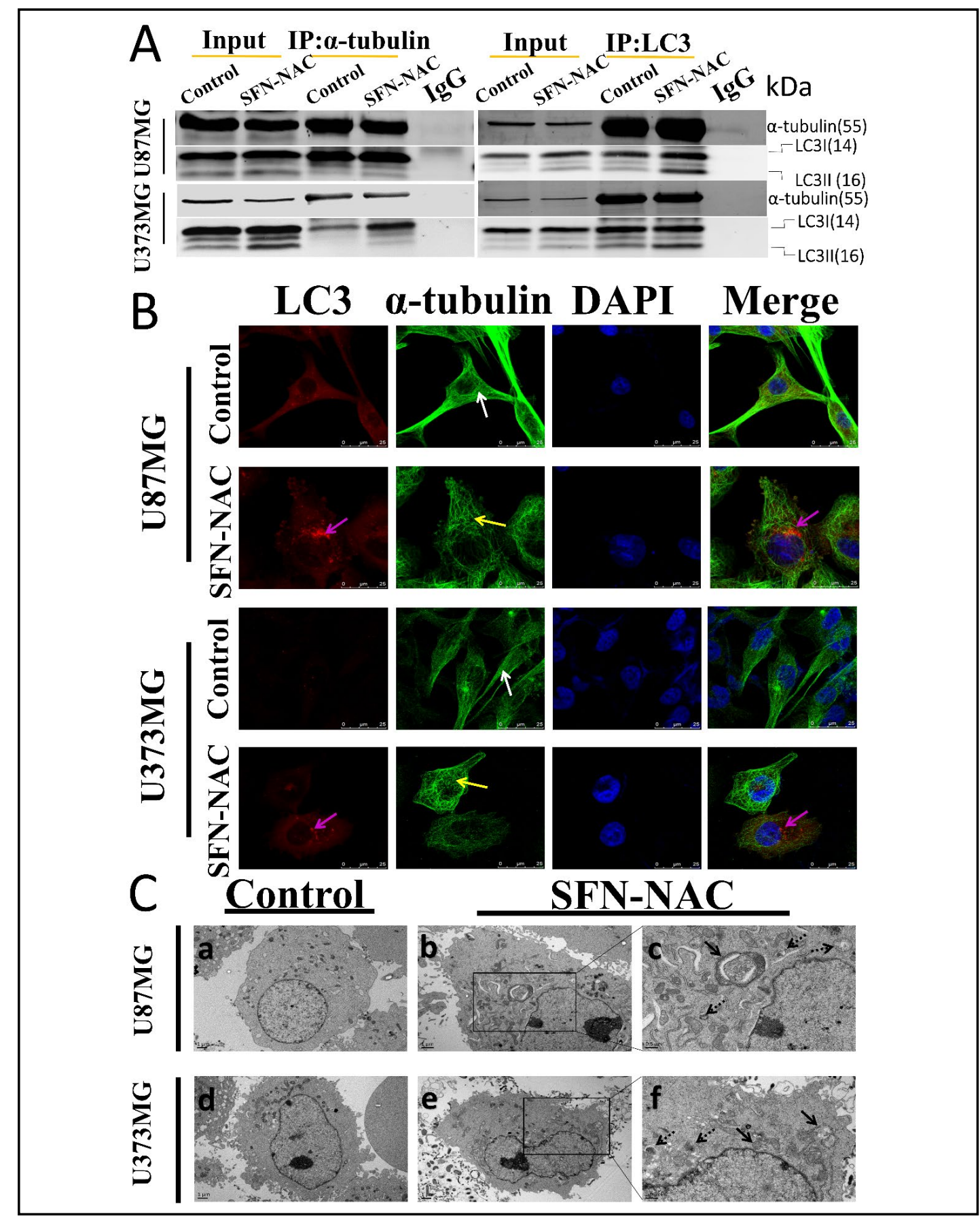

Fig. 4. SFN-NAC induced autophagy, downregulated $\alpha$-tubulin, and promoted the interaction between $\alpha$-tubulin and LC3 in U87MG and U373MG cells. U87MG and U373MG cells were treated with SFN-NAC $(0 \mu \mathrm{M}$ or $30 \mu \mathrm{M})$ for $24 \mathrm{~h}$. (A) Forward and reverse co-immunoprecipitation was employed to detect the interaction of $\alpha$-tubulin and LC3, and immunoprecipitation with normal mouse or rabbit IgG was used as the negative control. (B) Immunofluorescence staining of LC3 (red) and $\alpha$-tubulin (green) showed the increasing colocalization in the cells and the changes in microtubule morphology following treatment with SFN-NAC. Blue, DAPI-stained DNA; white arrows, normal microtubules; yellow arrows, abnormal microtubules; purple arrows, LC3 punctuation colocalized with $\alpha$-tubulin; scale bar, $25 \mu \mathrm{m}$. (C) Autophagosomes and autophagolysosomes were observed using transmission electron microscopy. Solid arrowheads mark double-membrane vesicle-forming autophagosomes and dotted arrowheads indicate single-membrane autolysosomes. Scale bars: a-b and d-e, $1 \mu \mathrm{m}$; c and f, $0.5 \mu \mathrm{m}$. 


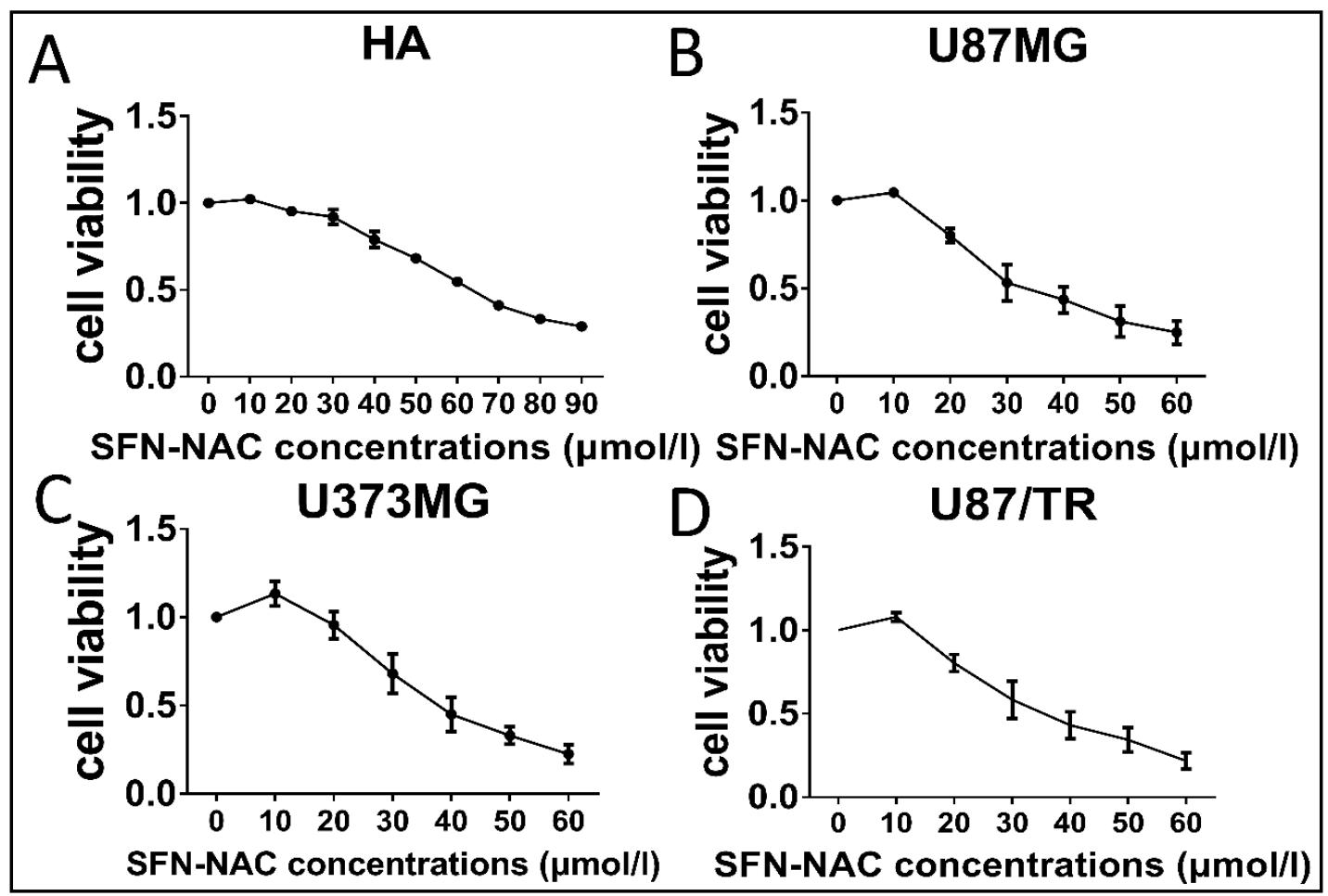

Fig. 5. Cell viability analysis after treatment with SFN-NAC. (A) HA, (B) U87MG, (C) U373MG, and (D) U87/ TR cells were treated with various concentrations of SFN-NAC $(0,10,20,30,40,50,60,70,80$, and $90 \mu \mathrm{M}$ or $0,10,20,30,40,50$, and $60 \mu \mathrm{M}$ ) for $24 \mathrm{~h}$. SFN-NAC significantly inhibited the proliferation of U87MG, U373MG, and U87/TR cells in a dose-dependent manner.

D). The $\mathrm{IC}_{50}$ value of the HA cells was approximately double that of other glioma cells. For all three types of glioma cells almost all cells died when treated with SFN-NAC at doses greater than $40 \mu \mathrm{M}$ for $24 \mathrm{~h}$. Treatment with SFN-NAC concentrations higher than approximately 80 $\mu \mathrm{M}$ for $24 \mathrm{~h}$ killed nearly all HA cells. These results demonstrated that SFN-NAC decreased the viability of glioma cells, including chemotherapy-resistant glioma cells, in a concentrationdependent manner. Most importantly, SFN-NAC exhibited low toxicity to normal human brain cells.

\section{Discussion}

A core therapeutic strategy of oncological pharmacology is to disrupt the integrity of spindle microtubules during mitosis of cancer cells, thereby preventing and limiting cell mitosis. Previous studies have indicated that SFN can inhibit cell growth and induce cell apoptosis by targeting microtubules in multiple tumor cells [33, 34]. Here, we have taken advantage of clinical information to identify $\alpha$-tubulin as a key prognosis factor in patients with gliomas and have elucidated that SFN-NAC may downregulate $\alpha$-tubulin through autophagy via phosphorylation of ERK1/2, thus inducing cell-cycle arrest and inhibiting cell growth in glioma cells (Fig. 6). It should be noted that there are concerns with the origin of the U87 cell line, but it is still considered a glioma cell line [35].

Studies of the bioavailability of bioactive compounds from crucifers have focused on glucoraphanin [36], the main glucosinolates in broccoli sprouts, and its bioactive isothiocyanate SFN, which is ultimately metabolized in the liver to form $\mathrm{N}$-acetyl-L-cysteine (-NAC). SFN-NAC has been established as a biomarker of crucifer consumption. It has been shown that SFN-NAC exhibits a longer half-life and a higher concentration in brain tissue compared with those of SFN [23], revealing that SFN-NAC may be more able to penetrate the 


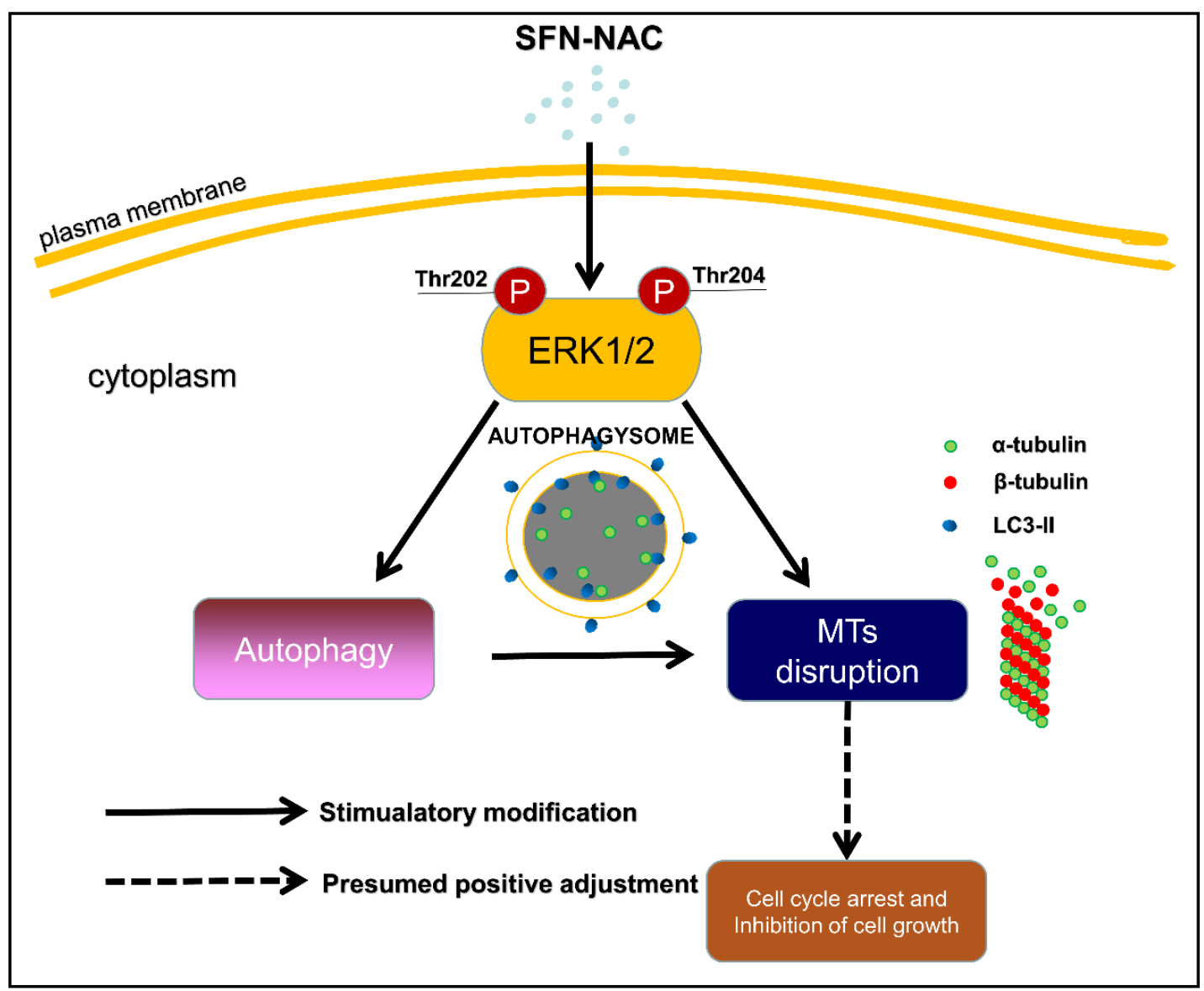

Fig. 6. Assumed signaling map for SFN-NAC-mediated inhibition of cell growth in human glioma cells.

blood-brain barrier to kill cancer cells [37].

In recent years, an increasing collection of genes have been found to be associated with survival in glioma patients [38]. Here, we demonstrated that increased expression of TUBA1B is associated with the grade of malignancy and predicted worse survival in glioma patients. The TUBA1B gene encodes the tubulin alpha-1B chain, an $\alpha$-tubulin subtype. Therefore, $\alpha$-tubulin might serve as a therapeutic targethttps://en.wikipedia.org/wiki/TUBA1B - cite note-pmid12054644-5 in cancer treatment, and targeting cell cycle progression is regarded as an effective strategy for cancer therapy. Mi et al. found that SFN covalently binds to tubulin, causes tubulin disruption, and induces growth arrest and apoptosis in A549 cells [39]. In another study, Zhou et al. showed that SFN metabolites could downregulate $\alpha$-tubulin via ERK1/2 activation, which directly interacts with $\alpha$-tubulin in lung cancer cells [40]. Here, we demonstrated that SFN-NAC downregulates $\alpha$-tubulin by phosphorylating ERK1/ 2 in glioma cells, which is consistent with results for other types of cancer cells. We also found that SFNNAC induced cell-cycle arrest.

ERK1/2, the major kinase among signaling molecules involved in the mitogenactivated protein kinase (MAPK) pathway, is activated via phosphorylation and is repressed by its specific phosphatases. The MAPK signaling cascade, including ERK1/2, transmits extracellular stimuli into cells and controls various aspects of cell physiology. Recent studies have shown that SFN markedly increased the phosphorylation of ERK1/2 in certain cancer cell lines. Some studies have found close correlations of the ERK1/2 signal pathway with cell division, invasion, and apoptosis. Our study has indicated that the agent induced autophagy via ERK1/2 in glioma cells treated with SFN-NAC.

\section{KARGER}


Autophagy is a highly conserved mechanism for maintaining homeostasis; it removes damaged or misfolded proteins and dysfunctional cellular organelles and enables cellular components to be recycled in cells ranging from yeast cells to mammalian cells. LC3, a marker of autophagy, plays a key role in autophagy. There are two forms of LC3 in cells: cytosolic LC3-I, which is located in the cytoplasm, and LC3-II, which is specifically localized on the membrane of autophagosomes. LC3-I is cleaved by a protease to expose a C-terminal glycine residue and is then conjugated to phosphatidylethanolamine to generate LC3-II. The cytosolic LC3 ratio (LC3-II/LC3-I ratio) is considered as a quantitative index of autophagy [41]. Through immunofluorescence confocal microscopy and co-immunoprecipitation experiments, we found that LC3 colocalizes and interacts with $\alpha$-tubulin in SFN-NACtreated cells, which confirmed the direct association of autophagosomes with microtubules. Meanwhile, western blot analysis showed that $\alpha$-tubulin was downregulated by SFN-NAC, which was mediated by autophagy. Taken together, these data suggested that SFN-NAC downregulated $\alpha$-tubulin through autophagy via phosphorylation of ERK1/2, which has not been previously reported to our knowledge.

Epidemiology studies have shown that ITC compounds, which are enriched in cruciferous vegetables, have a wide range of clinical applications and have been widely confirmed to exhibit carcinogenesis-inhibiting activity both in vitro and in vivo. ITC compounds have been demonstrated to additionally exert cytotoxic and antiproliferative effects in the micromolar range in a dose- and time-dependent manner in several cancer cell lines, including breast cancer, neuroblastoma, leukemia, prostate cancer, bladder cancer, lung cancer, and colorectal cancer [42]. These observations indicate the potential use of ITC compounds as anticancer agents. It is believed that ITC compounds play key roles in inhibiting cancer, motivating their potential clinical use, including in combination therapies. Recently, Bijangi et al. made an encouraging discovery that sulforaphane reduces the survival of GBM cells, glioblastoma stem-cell-like spheroids, and tumor xenografts through multiple cell signaling pathways and that this treatment does not affect the survival of normal human brain cells or normal human MSCs in both the cellular and animal experiments [43]. This study helps us better understand the mechanism of action of SFN and its analogs in tumor cells and can guides future clinical applications that utilize the degradation of tubulin by SFN analogs.

\section{Acknowledgements}

This work was supported by the National Natural Science Foundation of China (Grant number 31471145).

\section{Disclosure Statement}

The authors of this study declare no financial or commercial conflicts of interests.

\section{References}

-1 Dolecek TA, Propp JM, Stroup NE, Kruchko C: CBTRUS statistical report: primary brain and central nervous system tumors diagnosed in the United States in 2005-2009. Neuro Oncol 2012;14 Suppl 5:v1-49.

2 Roitberg B: Chemotherapy for glioblastoma multiforme (GBM). Surg Neurol 2006;65:107-108.

- 3 St-Coeur PD, Poitras JJ, Cuperlovic-Culf M, Touaibia M, Morin P, Jr.: Investigating a signature of temozolomide resistance in GBM cell lines using metabolomics. J Neurooncol 2015;125:91-102.

4 Zhang J, Zhang C, Cheng Y, Qi L, Wang S, Hou X: Microtubule and male sterility in a gene-cytoplasmic male sterile line of non-heading Chinese cabbage. J Sci Food Agric 2012;92:3046-3054.

5 Fujiwara K, Tilney LG: Substructural analysis of the microtubule and its polymorphic forms. Ann N Y Acad Sci 1975;253:27-50. 


\section{Cellular Physiology Cell Physiol Biochem 2018;51:528-542

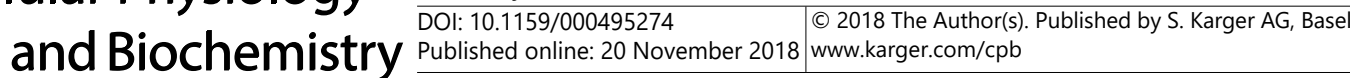

Liu et al.: Sulforaphane-N-Acetyl-Cysteine and $\alpha$-Tubulin

6 Syred HM, Welburn J, Rappsilber J, Ohkura H: Cell cycle regulation of microtubule interactomes: multilayered regulation is critical for the interphase/mitosis transition. Mol Cell Proteomics 2013;12:31353147.

7 Kotaja N, Macho B, Sassone-Corsi P: Microtubule-independent and protein kinase A-mediated function of kinesin KIF17b controls the intracellular transport of activator of CREM in testis (ACT). J Biol Chem 2005;280:31739-31745.

8 Handel MA, Roth LE: Cell shape and morphology of the neural tube: implications for microtubule function. Dev Biol 1971;25:78-95.

-9 Cassimeris L: Cell division: eg'ing on microtubule flux. Curr Biol 2004;14:R1000-1002.

10 Alesi GN, Jin L, Li D, Magliocca KR, Kang Y, Chen ZG, Shin DM, Khuri FR, Kang S: RSK2 signals through stathmin to promote microtubule dynamics and tumor metastasis. Oncogene 2016;35:5412-5421.

11 Hall JL, Cowan NJ: Structural features and restricted expression of a human alpha-tubulin gene. Nucleic Acids Res 1985;13:207-223.

12 Wang J, Chen W, Wei W, Lou J: Oncogene TUBA1C promotes migration and proliferation in hepatocellular carcinoma and predicts a poor prognosis. Oncotarget 2017;8:96215-96224.

13 Pensato V, Tiloca C, Corrado L, Bertolin C, Sardone V, Del Bo R, Calini D, Mandrioli J, Lauria G, Mazzini L, Querin G, Ceroni M, Cantello R, Corti S, Castellotti B, Solda G, Duga S, Comi GP, Cereda C, Soraru G et al.: TUBA4A gene analysis in sporadic amyotrophic lateral sclerosis: identification of novel mutations. J Neurol 2015;262:1376-1378.

14 Yokoi S, Ishihara N, Miya F, Tsutsumi M, Yanagihara I, Fujita N, Yamamoto H, Kato M, Okamoto N, Tsunoda T, Yamasaki M, Kanemura Y, Kosaki K, Kojima S, Saitoh S, Kurahashi H, Natsume J: TUBA1A mutation can cause a hydranencephaly-like severe form of cortical dysgenesis. Sci Rep 2015;5:15165.

15 Myers KA, Bello-Espinosa LE, Kherani A, Wei XC, Innes AM: TUBA1A Mutation Associated With Eye Abnormalities in Addition to Brain Malformation. Pediatr Neurol 2015;53:442-444.

-16 Lu C, Zhang J, He S, Wan C, Shan A, Wang Y, Yu L, Liu G, Chen K, Shi J, Zhang Y, Ni R: Increased alphatubulin $1 \mathrm{~b}$ expression indicates poor prognosis and resistance to chemotherapy in hepatocellular carcinoma. Dig Dis Sci 2013;58:2713-2720.

17 Lin K, Yang R, Zheng Z, Zhou Y, Geng Y, Hu Y, Wu S, Wu W: Sulforaphane-cysteine-induced apoptosis via phosphorylated ERK1/2-mediated maspin pathway in human non-small cell lung cancer cells. Cell Death Discov 2017;3:17025.

18 Arcidiacono P, Ragonese F, Stabile A, Pistilli A, Kuligina E, Rende M, Bottoni U, Calvieri S, Crisanti A, Spaccapelo R: Antitumor activity and expression profiles of genes induced by sulforaphane in human melanoma cells. Eur J Nutr 2017;10.1007/s00394-017-1527-7

19 Bessler H, Djaldetti M: Broccoli and human health: immunomodulatory effect of sulforaphane in a model of colon cancer. Int J Food Sci Nutr 2018;10.1080/09637486.2018.14399011-8.

-20 Bose C, Awasthi S, Sharma R, Benes H, Hauer-Jensen M, Boerma M, Singh SP: Sulforaphane potentiates anticancer effects of doxorubicin and attenuates its cardiotoxicity in a breast cancer model. PLoS One 2018;13:e0193918.

21 Kim SC, Choi B, Kwon Y: Thiol-reducing agents prevent sulforaphane-induced growth inhibition in ovarian cancer cells. Food Nutr Res 2017;61:1368321.

-22 Budnowski J, Hanschen FS, Lehmann C, Haack M, Brigelius-Flohe R, Kroh LW, Blaut M, Rohn S, Hanske L: A derivatization method for the simultaneous detection of glucosinolates and isothiocyanates in biological samples. Anal Biochem 2013;441:199-207.

-23 Wang H, Lin W, Shen G, Khor TO, Nomeir AA, Kong AN: Development and validation of an LC-MS-MS method for the simultaneous determination of sulforaphane and its metabolites in rat plasma and its application in pharmacokinetic studies. J Chromatogr Sci 2011;49:801-806.

24 Ma LL, Xing GP, Yu Y, Liang H, Yu TX, Zheng WH, Lai TB: Sulforaphane exerts neuroprotective effects via suppression of the inflammatory response in a rat model of focal cerebral ischemia. Int J Clin Exp Med 2015;8:17811-17817.

25 Jackson SJ, Singletary KW, Venema RC: Sulforaphane suppresses angiogenesis and disrupts endothelial mitotic progression and microtubule polymerization. Vascul Pharmacol 2007;46:77-84.

-26 Jackson SJ, Singletary KW: Sulforaphane inhibits human MCF-7 mammary cancer cell mitotic progression and tubulin polymerization. J Nutr 2004;134:2229-2236. 


\section{Cellular Physiology Cell Physiol Biochem 2018;51:528-542

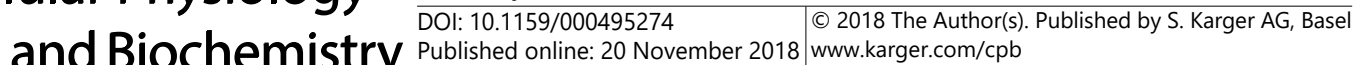

27 Mi L, Gan N, Cheema A, Dakshanamurthy S, Wang X, Yang DC, Chung FL: Cancer preventive isothiocyanates induce selective degradation of cellular alpha- and beta-tubulins by proteasomes. J Biol Chem 2009;284:17039-17051.

28 Mi L, Xiao Z, Hood BL, Dakshanamurthy S, Wang X, Govind S, Conrads TP, Veenstra TD, Chung FL: Covalent binding to tubulin by isothiocyanates. A mechanism of cell growth arrest and apoptosis. J Biol Chem 2008;283:22136-22146.

-29 Ohsumi Y: Historical landmarks of autophagy research. Cell Res 2014;24:9-23.

-30 Baust JM, Rabin Y, Polascik TJ, Santucci KL, Snyder KK, Van Buskirk RG, Baust JG: Defeating Cancers' Adaptive Defensive Strategies Using Thermal Therapies: Examining Cancer's Therapeutic Resistance, Ablative, and Computational Modeling Strategies as a means for Improving Therapeutic Outcome. Technol Cancer Res Treat 2018;17:1533033818762207.

-31 Fulda S: Targeting autophagy for the treatment of cancer. Biol Chem 2018;10.1515/hsz-2018-0105

32 Hu L, Wang H, Huang L, Zhao Y, Wang J: The Protective Roles of ROS-Mediated Mitophagy on (125)I Seeds Radiation Induced Cell Death in HCT116 Cells. Oxid Med Cell Longev 2016;2016:9460462.

33 Azarenko O, Okouneva T, Singletary KW, Jordan MA, Wilson L: Suppression of microtubule dynamic instability and turnover in MCF7 breast cancer cells by sulforaphane. Carcinogenesis 2008;29:2360-2368.

34 Oh CJ, Kim JY, Min AK, Park KG, Harris RA, Kim HJ, Lee IK: Sulforaphane attenuates hepatic fibrosis via NFE2-related factor 2-mediated inhibition of transforming growth factor-beta/Smad signaling. Free Radic Biol Med 2012;52:671-682.

35 Allen M, Bjerke M, Edlund H, Nelander S, Westermark B: Origin of the U87MG glioma cell line: Good news and bad news. Sci Transl Med 2016;8:354re353.

-36 Conzatti A, Froes FC, Schweigert Perry ID, Souza CG: Clinical and molecular evidence of the consumption of broccoli, glucoraphanin and sulforaphane in humans. Nutr Hosp 2014;31:559-569.

-37 Giacoppo S, Galuppo M, Iori R, De Nicola GR, Bramanti P, Mazzon E: The protective effects of bioactive (RS)glucoraphanin on the permeability of the mice blood-brain barrier following experimental autoimmune encephalomyelitis. Eur Rev Med Pharmacol Sci 2014;18:194-204.

-38 Zhang L, Liu Y, Wang M, Wu Z, Li N, Zhang J, Yang C: EZH2-, CHD4-, and IDH-linked epigenetic perturbation and its association with survival in glioma patients. J Mol Cell Biol 2017;9:477-488.

39 Mi L, Wang X, Govind S, Hood BL, Veenstra TD, Conrads TP, Saha DT, Goldman R, Chung FL: The role of protein binding in induction of apoptosis by phenethyl isothiocyanate and sulforaphane in human nonsmall lung cancer cells. Cancer Res 2007;67:6409-6416.

40 Zhou Y, Yang G, Tian H, Hu Y, Wu S, Geng Y, Lin K, Wu W: Sulforaphane metabolites cause apoptosis via microtubule disruption in cancer. Endocr Relat Cancer 2018;25:255-268.

41 Yu ZQ Ni T, Hong B, Wang HY, Jiang FJ, Zou S, Chen Y, Zheng XL, Klionsky DJ, Liang Y, Xie Z: Dual roles of Atg8-PE deconjugation by Atg4 in autophagy. Autophagy 2012;8:883-892.

42 Martin SL, Royston KJ, Tollefsbol TO: The Role of Non-coding RNAs and Isothiocyanates in Cancer. Mol Nutr Food Res 2018;10.1002/mnfr.201700913

43 Bijangi-Vishehsaraei K, Reza Saadatzadeh M, Wang H, Nguyen A, Kamocka MM, Cai W, Cohen-Gadol AA, Halum SL, Sarkaria JN, Pollok KE, Safa AR: Sulforaphane suppresses the growth of glioblastoma cells, glioblastoma stem cell-like spheroids, and tumor xenografts through multiple cell signaling pathways. J Neurosurg 2017;127:1219-1230. 